


\title{
La traducción de las ideas globales en la gobernanza de la educación secundaria en Colombia ${ }^{1}$
}

\author{
Claudia Milena Diaz Rios ${ }^{2}$ \\ McMaster University, Hamilton (ON), Canadá \\ diazricm@mcmaster.ca

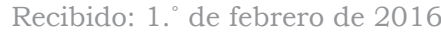 \\ Aceptado: 13 de junio de 2016 \\ Disponible en línea: 20 de diciembre de 2016
}

1 Este texto corresponde a un artículo de investigación que presenta parte de los resultados de la tesis doctoral de la autora, desarrollada entre los años 2012 y 2016. El estudio fue financiado por Colciencias (a través de la beca doctoral Francisco José de Caldas), McMaster University y el Consejo de Investigación en Ciencias Sociales y Humanidades de Canadá, por intermedio de una beca de investigación de la asesora de tesis, Dra. Michelle Dion.

2 Magister en Ciencias Sociales con Orientación en Educación, FLACSO (Argentina). Candidata a doctor en Ciencia Política, Departamento de Ciencia Política, McMaster University (Canadá). 


\title{
La traducción de las ideas globales en la gobernanza de la educación secundaria en Colombia
}

\section{Resumen}

Recientes reformas educativas en Colombia siguen una tendencia global en la que coinciden la descentralización curricular y la privatización de la educación, con procesos de centralización a través de exámenes estandarizados. Las teorias de la difusión sugieren que este cambio es el producto de la diseminación global de ideas de la Nueva Administración Pública, que son copiadas por tomadores de decisión a nivel doméstico. Sin embargo, a través de un análisis histórico comparativo, este estudio muestra que Colombia no copió las recomendaciones internacionales, sino que las tradujo para acomodar prácticas domésticas. Esta traducción es definida por el grado de influencia que tienen distintos grupos con intereses en la educación, a través de su capacidad de organización, su cercanía a la toma de decisiones, y el impacto público de los recursos que movilizan.

Palabras clave: educación secundaria; política educativa; difusión de políticas

\section{Translation of Global Ideas in Colombia's Secondary Education Governance}

\begin{abstract}
Recent education reforms in Colombia follow a global trend characterized both by curricular decentralization and privatization of education, as well as centralization processes through standardized tests. Diffusion theories suggest that this change has resulted from the global dissemination of New Public Management ideas replicated locally by policy makers. Through a comparative historical analysis, this study finds, however, that Colombia has not replicated foreign policy prescriptions, but translated them to accommodate local institutional practices. This translation is shaped by the influence of different interest groups associated with education policy, and specifically through their organizational capacity, their closeness to decision-making process, and the public impact of their power resources. Keywords: secondary education; education policy; policy dissemination
\end{abstract}

\section{Tradução das ideias globais na governança do ensino médio na Colômbia}

\section{Resumo}

Reformas educacionais recentes na Colômbia seguem uma tendência global em que coincidem descentralização curricular e privatização da educação, com processos de centralização através de testes padronizados. Teorias de difusão sugerem que esta mudança é produto da disseminação global de ideias da Nova Gestão Pública, que são copiados pelos tomadores de decisão no nível doméstico. No entanto, através de uma análise histórica comparativa, este estudo mostra que a Colômbia não copiou as recomendações internacionais, mas que traduziu para acomodar práticas domésticas. Esta tradução é definida pelo grau de influência de diferentes grupos de interesse na educação, através da sua capacidade de organização, sua proximidade com a tomada de decisão, bem como o impacto público de recursos mobilizados.

Palavras-chave: ensino médio; política educacional; difusão de politicas 


\section{Introducción}

En las últimas décadas, los países de América Latina han experimentado cambios importantes en el gobierno de la educación secundaria. Este estudio se enfoca particularmente en tres de ellos: 1) el paso de una educación homogénea y selectiva a una educación masiva que brinda diversas alternativas a los estudiantes; 2) la transición de un currículo centralizado a una relativa autonomía curricular; y 3) el desplazamiento parcial de la evaluación de los estudiantes por parte de los docentes, dado el protagonismo de los exámenes nacionales estandarizados. Basados en teorias de la difusión, distintos estudios han señalado que estas transformaciones en el gobierno de la educación son un efecto de medidas basadas en los principios de la Nueva Administración Pública (NAP), difundidas desde escenarios globales (McNeely, 1995; Whitty, 2000).

La literatura de la difusión de políticas tiene como base el argumento de que las decisiones nacionales son orientadas por fuerzas foráneas. Igualmente, se sostiene que dicha influencia externa a su vez produce convergencia en las políticas a nivel global, en la medida en que los actores domésticos copian prácticas de otros contextos (Dobbin, Simmons y Garrett, 2007). A pesar de compartir tal argumento, estas teorias proponen distintos mecanismos para explicar la difusión. Algunos trabajos emplean la noción de coerción para describir la forma en la que actores transnacionales hegemónicos imponen ciertas políticas a países relativamente dependientes (Arnove, Torres, Franz y Morse, 1996; Samoff, 2007).Otros argumentan que las políticas no son impuestas sino difundidas como normas o estándares adecuados para resolver asuntos públicos (Finnemore, 1993; Meyer, 1997; Schofer y Meyer, 2005). Finalmente, otros estudios muestran que la difusión se hace a través de mecanismos cognitivos, tales como redes de expertos que comparten y transmiten "soluciones" basadas en la evidencia de que son efectivas como política pública (Stone, 2004).

Ciertamente, los cambios en la educación secundaria en Colombia han sido motivados por mecanismos de difusión coercitivos, normativos y cognitivos. No obstante, una mirada detallada deja entrever 
ciertos desvíos respecto a las ideas globales. La educación secundaria en Colombia se ha masificado y el sector privado mantiene una participación considerable en la oferta educativa. Sin embargo, los estudiantes que asisten a las escuelas públicas tienen poca posibilidad de elegir su escuela. Igualmente, la autonomía curricular formalmente otorgada, a través del proyecto educativo institucional (PEI), está comprometida por una ausencia de autonomía en la gestión escolar. Por último, aunque la prueba Saber 11 determina ampliamente los contenidos y objetivos de aprendizaje de la educación secundaria, estos exámenes no son todavía instrumentos formales de estímulo y sanción para escuelas, como sí sucede en países con verdaderos cuasimercados educativos. Teniendo en cuenta tales "desvíos", este artículo busca explicar a través de un análisis histórico comparativo, por qué ideas globales sobre la gobernanza de la educación -frecuentemente catalogadas como ideas neoliberales-, parecen ser "libremente" aplicadas $^{3}$ en el contexto colombiano.

El presente análisis somete a prueba y refina los argumentos de las teorias de la difusión. Para ello, en la siguiente sección se explica cómo el concepto de 'traducción' corrige la exagerada pasividad que la noción de 'copia' les atribuye a los actores domésticos, y muestra que la confrontación política de dichos actores reinterpreta y modifica las ideas globales (Sahlin, 2008). A continuación, se presenta el diseño metodológico, y se ilustran los argumentos del artículo a través del análisis comparativo de tres periodos de ideas globales: 1) planeamiento educativo; 2) ideas de la NAP; 3) ideas de 'Educación para Todos'. En cada uno de estos periodos, el gobierno de la educación secundaria en Colombia sufrió cambios al tenor de las recomendaciones internacionales, pero las formas en que estas fueron adoptadas variaron considerablemente. Las conclusiones resumen la manera en que los defensores y opositores de las ideas globales a nivel doméstico definen el grado en que estas son modificadas y adaptadas a las condiciones institucionales locales en cada uno de los periodos analizados.

Este argumento se inspira en la noción de 'neoliberalismo libremente aplicado' (neoliberalism liberally applied) de Steiner-Khamsi, Silova y Johnson (2006). 


\section{La noción de traducción como marco para entender procesos de difusión}

En contraste con las teorías de la difusión, que argumentan que las ideas foráneas constriñen lo que los tomadores de decisión perciben como efectivo y apropiado para resolver problemas públicos, el institucionalismo histórico señala que son los arreglos institucionales domésticos los que moldean las perspectivas de los decisores de política (Mahoney, 2000; Thelen, 2004). Esta escuela de pensamiento sugiere que las politicas son una construcción histórica que depende, por una parte, de las decisiones pasadas y sus rendimientos crecientes, y por otra, de la permanente controversia entre distintos grupos que sostienen el sistema educativo (Thelen, 2014). En este sentido, las reglas de juego definidas por una política influyen en la conformación de grupos de 'perdedores' y 'ganadores' de la misma, pero a la vez, las formas en que estos grupos se confrontan puede alterar las reglas de juego, de manera tal vez gradual pero en ocasiones significativa y, muchas veces, inesperada (Thelen, 2004).

Si admitimos la validez tanto de las teorías de la difusión como del institucionalismo histórico, podemos proponer dos posibles escenarios. Si las ideas globales no contradicen los arreglos domésticos, los tomadores de decisión no tendrán problemas para aceptar la influencia externa, y con ello incrementar la legitimidad de las políticas domésticas. Pero si, por el contrario, las ideas globales desafian las políticas locales, los decisores se verán atrapados entre dos visiones opuestas y conflictivas. En cualquiera de estos escenarios, la noción de traducción resulta útil para interpretar esta interacción entre lo global y lo doméstico. Se entiende aquí por traducción el proceso por el cual se combinan nuevas ideas políticas de fuentes externas con ideas ya institucionalizadas a nivel doméstico (Campbell, 2004; Sahlin, 2008). Así, mientras las ideas de la NAP ejercen presión sobre los decisores de política educativa en Colombia, grupos de interés a nivel doméstico también presionan a los tomadores de decisión con el fin de reproducir o modificar las reglas de juego, de formas que sean convenientes a sus intereses y valores. 
Con base en los postulados del institucionalismo histórico, se sugieren aquí tres factores que definen la incidencia que tiene un grupo de interés en la traducción de una idea global: 1) la capacidad de organización (número de miembros y su cohesión interna); 2) su cercanía al proceso de toma de decisiones, ya sea por mecanismos formales (e.g. participación en debates legislativos) o por canales informales (e.g. nexos personales o profesionales con los tomadores de decisión); 3) el impacto público de los recursos que el grupo moviliza, tales como huelgas, manifestaciones, resultados de investigación, etc. La asimetria de estos factores entre distintos grupos define el nivel de traducción. Así, una total conformidad con la idea global es el resultado de una amplia incidencia de quienes abogan por ella frente a opositores débiles. Si, por el contrario, ambos grupos tienen un nivel relativamente equitativo de influencia, la idea tendrá que ser transformada para acomodar los intereses domésticos en conflicto. Por último, si los defensores de la idea global son débiles, la misma puede ser descartada o su adopción puede ser solamente simulada. El análisis del caso colombiano ilustra algunas de estas confrontaciones entre lo global y lo doméstico.

\section{La traducción de ideas globales en Colombia}

Tradicionalmente, Colombia ha sido un receptor entusiasta de ayuda externa técnica y financiera para la formulación e implementación de políticas educativas. A mediados del siglo XX, Colombia recibía de organismos transnacionales una suma equivalente a la mitad de su inversión pública en educación, y el país se había constituido en laboratorio de innovaciones educativas de dichos organismos (Arnove, 1980). En épocas más recientes, aunque la ayuda financiera ha disminuido, Colombia sigue dando la bienvenida a la asistencia técnica en materia educativa. En estas condiciones, este país puede considerarse un caso crucial en el que los postulados de las teorias de la difusión deberian cumplirse, dada la fuerte dependencia de actores externos (Gerring, 2007). De igual forma, este caso permite comprender mejor 
los mecanismos concretos por los cuales ideas externas inciden en las decisiones de política educativa.

Este estudio emplea un análisis histórico-comparativo, con el fin de rastrear los procesos de construcción de política de educación que afectan al nivel secundario desde los años cincuenta. Aunque se trate de un solo caso, la investigación descansa en múltiples observaciones a través de una comparación diacrónica, lo que permite valorar distintas explicaciones y aspectos del proceso en un contexto de variables relativamente constantes (Gerring, 2007). Este análisis se basa en fuentes primarias tales como reportes de organismos gubernamentales y no-gubernamentales, a nivel nacional e internacional, y 25 entrevistas con actores domésticos y foráneos relacionados con las decisiones de politica educativa en Colombia, tales como funcionarios de organismos internacionales, funcionarios del Ministerio de Educación, líderes del sindicato docente, etc. También se emplearon otros estudios sobre reforma educativa en Colombia como fuente de información (e.g. Le Bot, 1971; Arnove, 1980; Hanson, 1986; Helg, 1998; López, 2001; Lowden, 2004; Miñana, 2006, 2010; Bocanegra, 2010; Martinez, Ramírez y Villarraga, 2011). Estos insumos permitieron identificar las condiciones necesarias y suficientes bajo las cuales una idea global puede ser totalmente emulada.

\section{Los pobres intentos de emulación de las ideas planeamiento de la educación en la educación secundaria colombiana}

A diferencia de lo que ocurrió en otros países como México o Argentina, la provisión de la educación secundaria en Colombia no estuvo originalmente a cargo del Estado. Actores privados, especialmente la Iglesia católica, dominaron este ámbito educativo a tal punto que, para principios del siglo XX, cerca del $70 \%$ de la cobertura en educación secundaria estaba a cargo de proveedores privados (Helg, 1987). De la misma forma, el Estado tuvo en general poco control sobre lo que las escuelas, ya fuera públicas o privadas, hacian a nivel subnacional. En los departamentos y municipios típicamente se hacía caso omiso de las políticas y lineamientos del Ministerio de Educación, y el 
presupuesto educativo se usaba con fines políticos y clientelistas (Le Bot, 1971; Hanson, 1986). En consecuencia, el Estado fue poco exitoso en obligar a las escuelas a seguir un modelo nacional, y en cambio, otros actores tuvieron amplio margen para tomar decisiones sobre escuelas financiadas con dineros públicos. Con ello, estos actores consolidaron poderosas organizaciones que defendian sus intereses de cualquier intervención estatal (Helg, 1998).

Es en este contexto en el que arriban al país las ideas globales del planeamiento educativo. Desde la década del cincuenta, distintos organismos internacionales cuestionaron el carácter elitista de la educación secundaria en América Latina, y recomendaron enfáticamente la expansión de este nivel educativo como un factor crucial para promover el desarrollo económico (Wolf, 2002; Martinez et al., 2011). No obstante, para este nuevo modelo de educación secundaria, el Estado seguía siendo el principal responsable de expandir la oferta y de planear el currículo, para que respondiera a estimaciones rigurosas de necesidades de capital humano (Coombs, 1970; The World Bank, 1974).

Las ideas del planeamiento de la educación y del capital humano fueron difundidas en Colombia a través de mecanismos coercitivos y normativos, principalmente. En el marco de la Alianza para el Progreso, la Agencia de Cooperación para el Desarrollo y el Banco Mundial (BM) proporcionaron sustanciales sumas que condicionaron distintas politicas educativas, particularmente, la construcción de colegios de educación media diversificada (Arnove, 1980; Helg, 1998). Además de estos mecanismos coercitivos, la Unesco también difundió las normas de planeamiento educativo, a través de las Conferencias de Ministros y el Proyecto Principal de la Educación, en los que Colombia participó activamente (Martinez et al., 2011). Finalmente, en comparación con otros países como Chile, la difusión cognitiva fue muy débil en Colombia, en la medida en que no hubo un amplio entrenamiento de técnicos de planeamiento, con excepción probablemente del área de currículo. La Unesco y la Organización de Estados Iberoamericanos entrenaron personal del Ministerio de Educación en técnicas de planeamiento educativo y curricular, que también fueron incorporadas a 
los programas de formación de maestros de las más importantes facultades de educación del país (Martinez et al., 2011; Entrevista asesor de Renovación Curricular, 2014).

No obstante, estos mecanismos de difusión resultaron insuficientes para que las ideas de planeamiento educativo fueran completamente emuladas en Colombia. Aunque las élites politicas respaldaron formalmente estas ideas globales, también abogaron por una presencia estatal reducida que brindara educación secundaria solo a aquellos que no pudieran pagar por ella (MEN, 1957; Helg, 1998). A la negativa de las élites de extender la acción del Estado sobre la escuela secundaria, se sumó la acción de organizaciones de proveedores privados que resistieron con éxito los tímidos intentos estatales de regular su quehacer (Hanson, 1986; Helg, 1987, 1998). Esta dinámica persistió en 1970, cuando el gobierno se abstuvo de nacionalizar la educación secundaria con el argumento de que su inversión ofrecería educación gratuita a las clases más favorecidas (MEN, 1971, p. 292). En 1975 se aprueba finalmente la nacionalización, pero dicha ley solo fue parcialmente aplicada, dado que se aprobó como una medida populista sin los estudios adecuados para medir la capacidad del Estado. Además, gobernadores y alcaldes se anticiparon a nombrar rápidamente nuevos docentes, lo cual terminó por agotar las posibilidades del gobierno nacional de cubrir los costos de la nacionalización (Hanson, 1986).

Una situación similar se presentó en el marco de la Renovación Curricular. Este proceso inició en 1975 y se extendió hasta 1984, con el objeto de elaborar una planeación central del currículo y los planes de estudio (Martinez et al., 2011). Aunque hubo importantes avances en lo concerniente a la educación primaria, para la enseñanza secundaria solo se llegaron a publicar unos ejemplares de los programas de grado sexto a noveno, hacia finales de los años ochenta, cuya implementación no fue exigida por ley (Molano, 2011). Paradójicamente, el plan de estudio terminó siendo regulado por el Examen de Estado, el cual era aplicado masivamente desde 1968, con un propósito distinto, el de seleccionar a los estudiantes para el ingreso a la universidad. Fue este examen y su posterior reflejo en el decreto 080 de 1974, y no un planeamiento central del currículo, lo que unificó medianamente 
lo que se enseñaba a nivel secundario, especialmente en la educación media (Entrevistas con dos asesores de la Renovación Curricular, 2014). Por supuesto, estos contenidos no tuvieron mayor relación con las necesidades de capital humano en el país.

La influencia más clara de las ideas del planeamiento educativo en la educación secundaria colombiana vino de la mano de los Institutos de Educación Media Diversificada (INEM). Estos colegios, financiados parcialmente con créditos del BM, se encontraban directamente a cargo del Ministerio de Educación, y la formulación de su currículo estaba centralizada bajo el paradigma del planeamiento educativo (Martinez et al., 2011). Sin embargo, los INEM nunca llegaron a expandirse según lo planeado inicialmente, y para 1982, estos colegios solo atendian a 56 mil estudiantes de los 80 mil prometidos por el Ministerio de Educación (Duque, 1989).

En resumen, aunque las ideas globales prescribieran que el Estado debía planear y expandir la oferta educativa sobre la base de estimaciones de recurso humano, la presión de organismos internacionales fue insuficiente para modificar la negativa de las élites a hacerse cargo de una escuela de dichas características. Al contrario, dichas élites prestaron mayor atención a sectores privados muy bien organizados e influyentes -entre ellos la Iglesia-, que se resistieron a dejarse regular por el Estado. En definitiva, el esfuerzo de Colombia por emular las ideas del planeamiento educativo en educación secundaria se limitó a la expansión de una oferta de baja calidad y contenidos aislados para los más pobres, y a la construcción de algunos INEM que no alcanzaron a consolidarse.

\section{La adopción incompleta de las ideas de la NAP}

La fuerte crisis económica de los años ochenta provocó cambios sustanciales en las ideas globales. Del planeamiento central se pasó a políticas de ajuste estructural, privatización y descentralización (Astiz y Wiseman, 2005). A partir de análisis de costo-beneficio, varios estudios desarrollados por economistas de la educación mostraron que 
la educación secundaria, y en especial la de enfoque técnico o vocacional, producía tasas de retorno más bajas en comparación con la educación primaria, lo que por ende la hacía menos deseable como inversión pública (Psacharopoulos y Loxley, 1985; The World Bank, 1995). En consecuencia, los organismos multilaterales de financiamiento como el BM concentraron su inversión en la educación primaria, y recomendaron compartir los costos de la educación secundaria con unidades subnacionales, comunidades locales y actores privados. Instrumentos como los bonos educativos (vouchers) se publicitaron como mecanismos para devolverles la elección educativa a los padres y hacer a las escuelas más responsables con las expectativas de los mismos (The World Bank, 1995). Igualmente, se recomendaba que las decisiones curriculares fueran delegadas a la instituciones escolares, con el objeto de diversificar las opciones para los estudiantes, y hacer a las escuelas más relevantes para sus comunidades (Cepal y Unesco, 1992; The World Bank, 1995).

Como en décadas anteriores, estas ideas fueron de nuevo diseminadas en el país a través de mecanismos coercitivos, normativos y cognitivos. En lo referido a presiones coercitivas, el BM descontinuó el financiamiento a la expansión de la educación media diversificada (Entrevista exdirector de Oficina del Banco Mundial en América Latina, 2014), y sus créditos para educación secundaria se concentraron en bonos educativos a través del Programa de Fomento a la Cobertura en Educación Secundaria (PACES). El BM también ejerció presiones normativas a través de investigaciones desarrolladas en conjunto con universidades colombianas, que mostraban las bajas tasas de retorno de la educación técnica y media diversificada (e.g. Psacharopoulos y Loxley, 1985; Vélez y Psacharopoulos, 1987). Más aun, en contraste con el periodo anterior, las ideas de mercado esta vez sí fueron fuertemente difundidas por mecanismos cognitivos. Para la década de los noventa, la Dirección Nacional de Planeación (DNP) ya era conducida por economistas formados en el exterior, bajo ideas de mercado en la educación (Uribe, 2014). Estos economistas prepararon, en conjunto con el $\mathrm{BM}$, el proyecto de crédito para PACES, que estaba fuertemente asociado al proyecto de reforma educativa de los años noventa (Montenegro, 1995). 
Ahora bien, a pesar de que su difusión operó por todos los mecanismos, y de que las élites nacionales apoyaban las ideas de la NAP, las mismas no fueron fielmente copiadas. Consecuencias imprevistas de decisiones anteriores transformaron las coaliciones que sustentaban el sistema educativo y comprometieron la influencia de las élites. Aunque la nacionalización de la educación secundaria fue inconclusa, la ampliación de la escuela pública en décadas pasadas permitió la consolidación y unificación de la Federación Colombiana de Educadores (Fecode), un robusto sindicato docente que se convirtió en un actor politico muy influyente (Bocanegra, 2010). En el ambiente de apertura democrática promovido por la Asamblea Constituyente, Fecode logró una participación directa en el diseño de la nueva ley de educación. Esta participación es la que finalmente evitó una total conformidad con las ideas globales de la época para producir, en cambio, una suerte de negociación entre los intereses de distintos actores.

Hay que anotar que, para ese entonces, Fecode tenía preferencias relativamente ambiguas frente a la reforma educativa. Por una parte, y como crítica a la perspectiva centralizada del planeamiento curricular de décadas anteriores, al interior del sindicato se gestó una nueva línea de pensamiento denominada Movimiento Pedagógico, que abogaba por la autonomía curricular de los docentes y las escuelas (Martinez et al., 2011; Entrevista exlíder de Fecode, 2014). Por otra, sin embargo, se consideraba que una autonomía escolar demasiado amplia -que, por ejemplo, permitiera la contratación directa de maestros-, amenazaría las condiciones laborales de los docentes y la cohesión de Fecode (Entrevista exlíder de Fecode, 2014). Adicionalmente, el sindicato también defendia la educación técnica en tanto la consideraba una alternativa relevante para los estudiantes más pobres (Entrevista exlíder de Fecode, 2014), pero sobre todo porque eliminarla afectaba la cohesión y membresía de Fecode.

Igualmente, el gobierno estaba dividido en sus preferencias. De un lado, el DNP y el Ministerio de Hacienda elaboraron un proyecto de ley de descentralización de los servicios educativos, que buscaba la transferencia fiscal de las escuelas a los municipios, la competencia entre escuelas públicas y privadas, y la cancelación de las escuelas técnicas 
(Montenegro, 1995). Del otro lado, el Ministerio de Educación se negaba a ceder por completo la autoridad sobre las instituciones educativas, dada la falta de capacidad técnica a niveles locales, pero más que nada, se resistía a contradecir las preferencias de los docentes, dada la fortaleza del gremio (Entrevista exministro de Educación, 2014). En consecuencia, el Ministerio de Educación y Fecode redactaron en conjunto la propuesta de la nueva ley de educación y, con amplias movilizaciones del gremio, forzaron a la DNP y a Hacienda a negociar una alternativa intermedia (Lowden, 2004; Entrevistas exlíder Fecode y exministro de Educación, 2014).

Finalmente, las ideas globales de mercado no fueron completamente emuladas. La reforma educativa de 1993 transfirió las escuelas, principalmente a los departamentos, antes que a los municipios, y evitó la competencia entre escuelas, limitando la participación de proveedores educativos privados a aquellas situaciones en las que los cupos de las escuelas públicas fueran insuficientes (véase Ley 60, 1993). En términos curriculares, el Ministerio conservó su facultad de establecer lineamientos, y solo una autonomía muy limitada fue transferida a las escuelas por medio del PEI. Dicha autonomía no fue respaldada por otros factores importantes para su implementación tales como capacitación, presupuestos escolares o decisiones sobre la planta docente (Astiz y Wiseman, 2005; Entrevista exlíder Fecode, 2014). Las escuelas técnicas tampoco fueron eliminadas, aunque su financiamiento se vio seriamente comprometido. La responsabilidad por la promoción del componente técnico se le transfirió parcialmente al Servicio Nacional de Aprendizaje (SENA) (Entrevista líder sindical SENA, 2014). Estas modificaciones a los planteamientos iniciales del DNP y Hacienda terminaron por modificar el programa PACES (The World Bank, 1993) y lo convirtieron gradualmente en un subsidio para fomentar la retención de estudiantes de enseñanza secundaria (Entrevista Viceministra de Educación, 2014).

En resumen, a pesar de que las ideas globales de mercado fueron más influyentes que las del planeamiento educativo, dado el apoyo de un sector del gobierno y la intensidad de sus mecanismos de difusión, las mismas no pudieron ser completamente emuladas gracias a la 
resistencia de nuevos actores políticos, particularmente del sindicato docente. Esta resistencia motivó una reinterpretación de las ideas de mercado para ser adaptadas a nivel doméstico.

\section{Una mayor emulación de las ideas de la NAP}

En las últimas décadas, las ideas globales siguen privilegiando la participación de actores privados en la educación y la descentralización. No obstante, con la defensa que el movimiento Educación para Todos (EPT) ha hecho de la educación de calidad como derecho, y la ampliación del concepto de educación básica más allá de la enseñanza primaria, la educación secundaria ha vuelto a entrar en la agenda de los organismos internacionales (Entrevista exoficial del BM, 2014). El BM ha matizado su defensa directa de mecanismos de mercado en la educación, y en cambio promueve alianzas público-privadas para fortalecer la calidad de la educación pública (Patrinos, Osorio y Guáqueta, 2009).

Igualmente, el debate más reciente sobre el rol del Estado en materia educativa ha puesto el énfasis en la evaluación. Aunque los exámenes estandarizados ya aparecían en las recomendaciones de los noventa (The World Bank, 1995), en las últimas décadas se ha puesto un particular énfasis en el uso de estas pruebas como mecanismos de rendición de cuentas por parte de las escuelas y en la responsabilidad de los gobiernos por definir estándares de calidad (Unesco, 2000; The World Bank, 2011). En la educación secundaria en particular, estos estándares tienen que ver con la postergación de la especialización vocacional hacia el último tramo de la secundaria o post-secundaria, y con el énfasis en habilidades genéricas que preparen simultáneamente para continuar estudios terciarios y plantear trayectorias laborales (Braslavsky, 2001; Unesco y Orealc, 2002; The World Bank, 2005).

La crisis económica de mediados de los años noventa en Colombia intensificó los mecanismos de difusión de las ideas de la NAP en el país. En el marco del programa de ajuste estructural, el Fondo Monetario Internacional y el BM indicaron la necesidad de aumentar la 
responsabilidad fiscal de municipios y departamentos en la provisión de la educación (The World Bank, 2008). En consonancia, el gobierno colombiano se comprometió a profundizar la descentralización transfiriendo escuelas a municipios, tal como pretendia hacerlo en la década anterior. Adicionalmente, el Banco Interamericano de Desarrollo aportó ayuda financiera para la definición de estándares curriculares y mecanismos de evaluación (véase IADB, 1999). Más recientemente, en 2012, el BM ofreció un préstamo y asistencia técnica con el objeto de reformar el currículo de la educación media y desestimular la educación técnica (Entrevistas exfuncionaria del Ministerio de Educación y oficial del BM en Colombia, 2014).

Además de estos mecanismos coercitivos, el BM también ejerció presiones normativas a través de la valoración positiva de diversas experiencias de alianzas público-privadas en el país que incluyen PACES, a pesar de su corta duración, la contratación con escuelas confesionales, y las escuelas concesionadas (e.g. Banco Mundial, 2006, 2008; Patrinos, Osorio y Guáqueta, 2009). El BM también incluyó consistentemente en sus recomendaciones al país la implementación de estándares curriculares y sistemas de medición de la calidad y rendición de cuentas, así como la participación en exámenes estandarizados internacionales que permitieran identificar metas nacionales de calidad (Banco Mundial, 2007, 2008). Así mismo, el diagnóstico del país en el marco de EPT se señalaba la necesidad de estándares curriculares para enfrentar la inequidad educativa y la falta de objetivos comunes de aprendizaje (MEN, 2000).

Por último, los mecanismos cognitivos incluyeron, por una parte, las comunidades de economistas entrenados en el exterior con ideas neoliberales que seguian siendo predominantes en la DNP y el Ministerio de Hacienda, y, por otra parte, los técnicos en evaluación entrenados también con la ayuda de exámenes internacionales como PISA, TIMMS, PIRLS y LLECE, que ayudaron a consolidar una cultura de la evaluación en el país (Entrevistas dos ex-directores del ICFES).

Aunque los mecanismos de difusión fueron similares a los de la década anterior, un cambio en las posiciones y relaciones de fuerza de 
los actores del sistema educativo hizo que la traducción de las ideas globales fuera esta vez más fiel. La crisis económica le dio impulso y legitimidad a medidas que propendian por reducir y hacer un mejor uso del gasto público (Exposición de motivos Ley 715, 2000; Lowden, 2004). En este contexto, el gobierno logró una mayor consistencia en sus intereses. El DNP y Hacienda seguían empeñados en profundizar la descentralización educativa y en crear mecanismos para estimular la eficiencia del gasto. Por su parte, el Ministerio de Educación compartía esta expectativa y esperaba además recuperar el control sobre las escuelas (Lowden, 2004; Entrevista exasesor Ministerio de Educación, 2014).

Esta administración movilizó distintas estrategias para incrementar la legitimidad de la reforma y disminuir los recursos de poder del sindicato docente. El gobierno nacional emprendió una campaña mediática que ponía como centro del debate el tema de la calidad (Miñana, 2006), y mostraba los bajos resultados educativos de las escuelas públicas pese al incremento del presupuesto educativo. Dicha campaña y la cohesión del gobierno en un contexto de crisis económica, neutralizaron el poder político de Fecode, dado que sus constantes demandas por el mejoramiento de sus condiciones laborales -aunque justas-, no fueron respaldadas por una opinión pública agobiada por el desempleo. Por ende, las movilizaciones fueron pobremente apoyadas por la ciudadanía (Entrevista exlíder Fecode, 2014) y menos aun por el Ministerio de Educación que, al contrario, las penalizó y enmarcó como politización de la educación (El Tiempo, 2002).

Todos estos elementos permitieron que se incorporaran cambios que no habian sido posibles en los años noventa. La reforma fiscal del año 2001 dio un giro desde un financiamiento sobre la base de los costos históricos de cada unidad subnacional, al pago por capitación o estudiante matriculado (Ley 715,2001 ). Aunque la competencia entre escuelas siguió por fuera de la legislación, la financiación a la demanda aumentó el incentivo para la contratación de servicios educativos privados por parte de las entidades territoriales, en la medida en que ello permitió pactar menores precios por estudiante matriculado (Entrevista exasesor Ministerio de Educación, 2014). Esta contratación 
también resultó atractiva para flexibilizar las condiciones laborales de los maestros, evitando su contratación en el marco del estatuto docente (Entrevista exministra de Educación, 2014). Entre los años 2002 y 2006, el porcentaje de matrícula de la educación contratada con respecto a la oficial pasó del 3 al 9\% (Castillo, 2008).

La reforma fiscal fue también la base para robustecer el sistema de evaluación. Además de avanzar en el diseño de estándares curriculares para la enseñanza secundaria, se aprovechó la fuerte tradición y legitimidad del Servicio Nacional de Pruebas (ICFES) y el Examen de Estado, para alinear los estándares a las pruebas de grado undécimo (Entrevistas dos exdirectores del ICFES, 2014). Con dicha alineación, el Estado logró centralizar el control de la educación secundaria, mientras que la responsabilidad por los resultados educativos sí permaneció descentralizada en las escuelas y entidades territoriales (Miñana, 2010). Por otra parte, aunque en Colombia las pruebas estandarizadas tradicionalmente han servido para establecer clasificaciones de escuelas según su rendimiento, su uso como instrumento para estimular o sancionar el desempeño de docentes y escuelas aún es relativamente limitado. Solo recientemente han empezado a surgir iniciativas para premiar rectores y docentes de instituciones que presenten progresos. De tal manera, la supuesta rendición de cuentas promovida por las ideas de la NAP es apenas incipiente en el país, posiblemente para evitar las respuestas negativas del gremio docente.

De otro lado, mientras la traducción de las ideas globales ligadas a la evaluación y la privatización fue cada vez más fiel, no sucedió lo mismo con la educación técnica secundaria. Aunque se esperaba que, con la transferencia al SENA del componente técnico, este tipo de educación fuera gradualmente desestimulado, esto no sucedió. A partir de la década del dos mil, como una estrategia para aumentar su cobertura, el SENA incentivó su asesoría a colegios oficiales para desarrollar un componente técnico (Entrevistas dos líderes sindicales y una ex-directora regional del SENA, y una funcionaria del Ministerio de Educación, 2014). Esta asesoria, llamada posteriormente "Articulación entre la educación media y la superior", se expandió descontroladamente y para el año 2011, 2.123 escuelas ya participaban de ella (Econometría 
Consultores, 2012). Esta expansión alimentó innumerables seguidores entre los rectores, docentes, padres y estudiantes. Esta popularidad abrió oportunidades de ganancias políticas a nivel departamental y municipal, con lo cual alcaldes, gobernadores y el mismo Ministerio se resistieron a desincentivarla (Entrevistas exfuncionaria del Ministerio de Educación y oficial del BM para Colombia, 2014). De esta forma, a pesar de la asistencia técnica y de las promesas de crédito del BM para reformar este tipo de educación, el Ministerio de Educación, conducido por quien anteriormente dirigiera el SENA, terminó por rechazar el crédito ofrecido por el BM y canceló el proceso de reforma iniciado en el año 2012 (Entrevista exfuncionaria Ministerio de Educación, 2014). En resumen, a diferencia de Fecode, los defensores de la educación técnica tuvieron mucha más cohesión y recursos de poder para rechazar las ideas globales de reforma a este tipo de educación secundaria.

\section{Conclusión}

Desde hace varias décadas, Colombia ha experimentado una fuerte difusión de ideas globales en educación, promovidas desde diversos organismos transnacionales. Si bien estas ideas han tenido una fuerte resonancia en la política educativa colombiana, la presión externa no ha sido determinante en las reformas educativas. En los años cincuenta y sesenta, la presión coercitiva y normativa de las ideas globales del planeamiento educativo fue insuficiente para vencer la resistencia de una élite política que, en una consistente alianza con la Iglesia, favoreció sus intereses en una mínima intervención del Estado en la educación secundaria. En consecuencia, las ideas del planeamiento educativo fueron solamente simuladas a través de una lenta expansión de la educación pública para los más pobres y el establecimiento de algunos colegios de educación media diversificada.

No obstante, la simulación del planeamiento educativo tuvo un efecto inesperado para futuras reformas. En la década de 1990, las ideas de la NAP fueron también difundidas por mecanismos coercitivos, normativos y cognitivos. En contraste con lo sucedido en los 
años sesenta, estas ideas gozaban del amplio soporte de un sector influyente del gobierno. Este apoyo, sin embargo, fue insuficiente para vencer la resistencia de un sindicato docente que se consolidó con la expansión de la escuela pública para los más pobres. La fuerte oposición del gremio docente equiparó la influencia de los defensores de las ideas de la NAP y comprometió la total conformidad con las mismas. En definitiva, el gobierno se vio forzado a acomodar los intereses de los docentes, a través de la prohibición de la competencia entre escuelas públicas y privadas, la limitación de la autonomía escolar, y el uso de exámenes estandarizados solo como mecanismo informativo de resultados sin consecuencias tangibles para escuelas o docentes.

Contrario a lo que ocurrió en la década de los noventa, las ideas de la NAP tuvieron mayor incidencia en la del dos mil. Esta vez, además de mecanismos de difusión coercitivos, normativos y cognitivos, y del amplio y cohesivo soporte del gobierno, Fecode vio disminuida su oposición. La crisis económica y los escasos resultados de la reforma educativa anterior ayudaron al gobierno a minar la legitimidad de las demandas del sindicato por mayores recursos. De tal forma, el gobierno estuvo en mayor capacidad de emular las ideas globales a través de la financiación a la demanda, la estandarización curricular, y el fortalecimiento de los mecanismos de evaluación. No obstante, aunque el sector privado tiene cada vez mayor participación en la provisión educativa, no hay verdadera competencia entre escuelas privadas y públicas. Así mismo, en los sistemas estandarizados de medición de aprendizajes de los estudiantes no han calado del todo instrumentos de sanción y estímulos, típicos de cuasimercados de la educación como Chile e Inglaterra.

La comparación de estos tres periodos históricos de reformas educativas muestra que las ideas globales y sus mecanismos de difusión son necesarios, pero no suficientes para determinar el contenido de las reformas. Igualmente, la relativa compatibilidad entre las ideas globales y los arreglos institucionales domésticos es también insuficiente para una total conformidad con las normas foráneas. La evidencia aquí analizada sugiere más bien una causalidad coyuntural, pues se requiere la combinación de tres factores para una completa 
emulación de las ideas globales: 1) difusión de las ideas; 2) apoyo a las ideas foráneas por grupos domésticos influyentes; 3) débil o nula oposición de actores domésticos. Esta causalidad rompe con la visión determinista sobre la influencia de ideas y actores transnacionales en la política doméstica, que en ocasiones asumen estudios de política educativa en la región (e.g. Arnove et al., 1996; Coraggio y Torres, 1997). Además, como la combinación de estos tres factores no ha sido necesariamente frecuente, la traducción de las ideas globales sobre educación en Colombia ha mostrado una dinámica de confrontación política en la que las decisiones se desvian de la norma global y producen consecuencias inesperadas para distintos grupos de actores. Estas consecuencias modifican la confrontación política doméstica y pueden comprometer o facilitar la posterior adopción de otras normas globales.

\section{Referencias}

Arnove, R. (1980). Education Policies in the National Front. En R. A. Berry and M. Solaun (Eds.), Politics of Compromise: Coalition Government in Colombia (pp. 381-411). New Brunswick, N.J: Transaction Publishers.

Arnove, R., Torres, A., Franz, S. and Morse, K. (1996). A Political Sociology of Education and Development in Latin America. International Journal of Comparative Sociology, 37(1-2), 140-158.

Astiz, M. and Wiseman, A. (2005). Slouching Toward a Global Ideology. Decentralization and devolution of Central Education Authority. En D. Baker and G. LeTendre (Eds.), National differences, global similarities: world culture and the future of schooling (pp. 134-149). Stanford, Calif.: Stanford Social Sciences.

Banco Mundial. (2006). Colombia: Contratación de Servicios Educativos. Bogotá: Banco Mundial.

Banco Mundial. (2007). Colombia 2006-2010: Una ventana de oportunidad. Notas de politicas presentadas por el Banco Mundial. Bogotá: BIRD - Banco Mundial. 
Banco Mundial. (2008). La calidad de la educación en Colombia: un análisis y algunas opciones para un programa de politica. Bogotá: Banco Mundial.

Bocanegra, H. (2010). Las politicas educativas y el magisterio colombiano en la década de los 80. Diálogo de Saberes, 32, 29-44.

Braslavsky, C. (2001). Educación secundaria ¿cambio o inmutabilidad? Buenos Aires: IIPE - Unesco - Santillana.

Campbell, J. L. (2004). Institutional Change and Globalization. New Jersey: Princeton University Press.

Castillo, A. (2008). ¿Calidad o Cobertura? Análisis de la Contratación de los Servicios Educativos en Colombia. Bogotá: Educación Compromiso de Todos Conversemos sobre Educación.

CEPAL y Unesco. (1992). Educación y conocimiento: eje de la transformación productiva con equidad. Santiago de Chile: CEPAL.

Coombs, P. (1970). What is Educational Planning? Paris: IIEP - Unesco.

Coraggio, J. L. y Torres, R. M. (1997). La educación según el Banco Mundial: un análisis de sus propuestas y métodos. Buenos Aires: Miño y Dávila.

Dobbin, F., Simmons, B. A. and Garrett, G. (2007). The Global Diffusion of Public Policies: Social Construction, Coercion, Competition, or Learning? Annual Review of Sociology, 33, 449-472.

Duque, H. (1989). Las Politicas de Descentralización del Sector Educativo Nacional, 1988-1989. Bogotá: Mimeografiado.

Econometría Consultores. (2012). Evaluación de la Estrategia de Articulación de la Educación Media con la Educación Superior y la Formación para el Trabajo (Informe Final). Bogota: DNP.

El Tiempo. (2002, junio 22). A despolitizar la educación. Recuperado de http:// www.eltiempo.com/archivo/documento/MAM-1339850 
Exposición de motivos Ley 715. (2000, agosto 1). Gaceta del Congreso, 294 [Bogotá, Colombia].

Finnemore, M. (1993). International Organizations as Teachers of Norms: The United Nations Educational, Scientific, and Cutural Organization and Science Policy. International Organization, 47(4), 565-597.

Gerring, J. (2007). Is There a (Viable) Crucial-Case Method? Comparative Political Studies, 4O(3), 231-253.

Hanson, M. (1986). Educational Reform and Administrative Development: The Cases of Colombia and Venezuela. Stanford: Hoover Press.

Helg, A. (1987). La educación en Colombia, 1918-1957: una historia social, económica y política. Bogotá: CEREC.

Helg, A. (1998). La Educación en Colombia 1958-1980. En A. Tirado, J. Jaramillo y J. Melo (Eds.), Nueva Historia de Colombia (Vol. IV, pp. 135-58). Bogotá: Planeta.

IADB. (1999). New School System Program: Reform of Education Management and Participation (No. Loan Proposal CO-0142). IADB. Recuperado de http:// www.iadb.org/en/projects / project-description-title, 1303.html?id=co0142\#doc

Le Bot, I. (1971). El sistema escolar colombiano. Bogotá: DANE.

Ley 60. (1993, agosto 12). Diario Oficial República de Colombia, 40.987 [Bogotá, Colombia].

Ley 715. (2001, agosto 1). Diario Oficial de la República de Colombia, 44.654 [Bogotá, Colombia].

López, M. M. (2001). Pluralidad en la manera de hacer politica educativa: reforma de descentralización de la educación. Bogotá: Fundación Corona.

Lowden, P. (2004). Education Reform in Colombia: The Elusive Quest for Effectiveness. En R. R. Kaufman and J. M. Nelson (Eds.), Crucial needs, weak 
incentives: social sector reform, democratization, and globalization in Latin America (pp. 315-349). Washington, D.C. - Baltimore: Woodrow Wilson Center Press - Johns Hopkins University Press.

Mahoney, J. (2000). Path dependence in historical sociology. Theory and Society, 29(4), 507-548.

Martinez, A., Ramírez, C. E. N. y Villarraga, J. O. C. (2011). Currículo y modernización: cuatro décadas de educación en Colombia. Bogotá: Cooperativa Editorial Magisterio.

McNeely, C. L. (1995). Prescribing National Education Policies: The Role of International Organizations. Comparative Education Review, 39(4), 483-507.

MEN (Ministerio de Educación Nacional). (1957). Informe del proyecto para el I plan quinquenal. Bogotá: MEN.

MEN (Ministerio de Educación Nacional). (1971). La Educación ante el Congreso. Bogotá: MEN.

MEN (Ministerio de Educación Nacional). (2000). Educación para todos: Evaluación en el año 2000. Informe Nacional - Colombia. Bogotá: Unesco.

Meyer, J. (1997). World Society and the Nation-State. The American Journal of Sociology, 103(1), 144-181.

Miñana, C. (2006). Estado, Mercado y Comunidad: Reformas Educativas y Redefinición de lo Público en Colombia (Tesis Doctoral). Universidad de Barcelona, Barcelona.

Miñana, C. (2010). Politicas neoliberales y neoinstitucionales en un marco constitucional adverso. Reformas educativas en Colombia 1991-2010. Propuesta Educativa, 19(2), 37-52.

Molano, M. (2011). Carlos Eduardo Vasco Uribe. Trayectoria biográfica de un intelectual colombiano: una mirada a las reformas curriculares en el país. Revista Colombiana de Educación, 61, 161-198. 
Montenegro, A. (1995). An Incomplete Reform: The Case of Colombia. Human Capital Development \& Operations Policy. Working Papers. The World Bank.

Patrinos, H., Osorio, F. B. and Guáqueta, J. (2009). The Role and Impact of Publicprivate Partnerships in Education. Washington D.C.: World Bank Publications.

Psacharopoulos, G. and Loxley, W. A. (1985). Diversified Secondary Education and Development: Evidence from Colombia and Tanzania. Baltimore: Johns Hopkins University Press - The World Bank.

Sahlin, K. (2008). Circulating Ideas: Imitation, Translation and Editing. In R. Greenwood, C. Oliver and R. Suddaby (Eds.), The SAGE Handbook of Organizational Institutionalism (pp. 218-242). London: SAGE.

Samoff, J. (2007). Institutionalizing International Influence. In R. Arnove and C. A. Torres (Eds.), Comparative Education: The Dialectic of the Global and the Local (pp. 47-78). Landham, MD: Rowman \& Littlefield Publishers.

Schofer, E. and Meyer, J. (2005). The Worldwide Expansion of Higher Education in the Twentieth Century. American Sociological Review, 70(6), 898-920.

Steiner-Khamsi, G., Silova, I., \& Johnson, E. M. (2006). Neoliberalism liberally applied. En World Yearbook of Education 2006: Education, Research and Policy: Steering the Knowledge-Based Economy (C. Díaz, Trad.) (pp. 217-245). London New York: Routledge.

Stone, D. (2004). Transfer agents and global networks in the "transnationalization" of policy. Journal of European Public Policy, 11(3), 545-566.

Thelen, K. (2004). How Institutions Evolve: The Political Economy of Skills in Germany, Britain, the United States, and Japan. Cambridge - New York: Cambridge University Press.

Thelen, K. (2014). Varieties of Liberalization and the New Politics of Social Solidarity. Cambridge - New York, N.Y: Cambridge University Press.

The World Bank. (1974). Education Sector. Working Paper. The World Bank. 
The World Bank. (1993). Secondary Education Project. Staff Apraisal Report 11834-CO. The World Bank. Recuperado de http://www-wds.worldbank.org/ external/default/WDSContentServer/WDSP/IB/1993/11/19/000009265_39 61004165109/Rendered/PDF/multi_page.pdf

The World Bank. (1995). Priorities and Strategies for Education. A World Bank Review. The World Bank.

The World Bank. (2005). Expanding Opportunities and Building Competencies for Young People: A New Agenda for Secondary Education. Washington, D.C.: The World Bank.

The World Bank. (2008). Colombia - Structural Fiscal Adjustment; and First, Second, and Third Programmatic Fiscal and Institutional Adjustment Loan Projects (No. 43357) (pp. 1-74). The World Bank. Recuperado de http://documents.worldbank.org/curated/en/2008/04/9581944/ colombia-structural-fiscal-adjustment-project-first-programmatic-fiscalinstitutional-adjustment-loan-second-programmatic-fiscal-institutionalstructural-adjustment-loan-third-programmatic-fiscal-institutional-structuraladjustment-loan-colombia-structural-fiscal-adjustment-project

The World Bank. (2011). Learning for all: investing in people's knowledge and skills to promote development. Washington D.C.: The World Bank.

Unesco. (2000). Dakar Framework for Education. Education for All: Meeting our Collective Comitments. París: Unesco.

Unesco y Orealc. (2002). ¿Qué educación secundaria para el siglo XXI? Santiago de Chile: Unesco - Orealc.

Uribe, C. (2014). The Positioning of Economic Rationality in Colombia. Bulletin of Latin American Research, 33(1), 46-59.

Vélez, E. and Psacharopoulos, G. (1987). The external efficiency of diversifie secondary schools in Colombia. Economics of Education Review, 6(2), 99-110. 
Whitty, G. (2000). Marketization and privatization in mass education systems. International Journal of Educational Development, 20(2), 93-107.

Wolf, A. (2002). Does education matter?: myths about education and economic growth. London: Penguin.

\section{Cómo citar este artículo}

Díaz Rios, C. M. (2017). La traducción de las ideas globales en la gobernanza de la educación secundaria en Colombia. Universitas Humanística, 83, 31-56. http:/ /dx.doi.org/10.11144/Javeriana.uh83.tigg 